
\title{
Research S Suare \\ The World Health Organisation's Caregiver Skills Training Programme for Autism and Developmental Disorders: Adapting and Pretesting in Hong Kong
}

\section{Paul W.C. Wong ( $\sim$ paulw@hku.hk)}

The University of Hong Kong, Hong Kong Special Administrative Region

Yan Yin Lam

The University of Hong Kong, Hong Kong Special Administrative Region

Janet Siu Lau

The University of Hong Kong, Hong Kong Special Administrative Region

Hung Kit Fok

The University of Hong Kong, Hong Kong Special Administrative Region

\section{Research Article}

Keywords: children with autism or developmental delays, Hong Kong, social service providers, service needs, Caregiver Skills Training Programme.

Posted Date: April 15th, 2021

DOl: https://doi.org/10.21203/rs.3.rs-408342/v1

License: (9) (1) This work is licensed under a Creative Commons Attribution 4.0 International License. Read Full License 


\section{Abstract}

Background The World Health Organisation Caregiver Skills Training programme (WHO-CST) was developed to strengthen a caregiver's skills in supporting their child's development and the caregiver's well-being. The WHO-CST Hong Kong (HK) was adapted to support families with children suspected of having autism spectrum disorder (ASD) while the families were waiting for a confirmed diagnosis.

Aim This sequential mixed methods study aimed to explore the adaptation process, using a formative qualitative method. It assessed the acceptability, feasibility, and perceived benefits of the WHO-CST, using caregivers' qualitative and quantitative prepost feedback.

Methods Data examined included (1) two consultation meetings with stakeholders; (2) a detailed review of translated and adapted WHO-CST HK materials by Master Trainees (MTs) $(n=10)$ trained by WHO representatives; (3) need assessment focus group interviews with caregivers $(n=15)$ of children with ASD; and (4) prepost CST programme qualitative focus group interviews and quantitative evaluation $(n=$ 11).

Results Consultation with stakeholders suggested that the programme is acceptable for the local community, but the home visit and fidelity components were initially considered to be challenges for the feasibility and sustainability of the programme. Thematic analyses of the focus group interview with caregivers generated six subthemes from preprogramme focus group interviews and five subthemes from postprogramme focus group interviews. The programme was reported to be helpful for caregivers' skills development and for aiding children's verbal and social skills development. There was also improvement in caregivers' mental health after completing the programme.

Conclusion The prepilot evaluation findings of WHO-CST HK indicate that it is feasible and acceptable to implement the programme in a metropolitan area where families have busy working schedules. The findings indicate potential programme developments for supporting families in communities in high income countries.

\section{Introduction}

The 2010 Global Burden of Disease study revealed that 52 million people have autism (1 in 132) and the World Health Organisation (WHO) estimates the international prevalence of autism spectrum disorder (ASD) is $0.76 \%{ }^{1}$. Hong Kong (HK) has a population of 7.5 million with approximately $16 \%(n=1,180,000)$ of people aged under 20 years old ${ }^{2}$. The number of children newly diagnosed with ASD has increased almost three times from 755 in 2006 to 2,021 in 2015. Autism spectrum disorder comprised over $60 \%$ of the caseload of the child and adolescent services in public hospitals in 2015-2016 3 .

The local government provides comprehensive support from early diagnosis and medical treatment to special education through Child Assessment Centres. It provides early identification and referral for further intensive services with the Social Welfare Department and Education Bureau providing special 
social and educational services ${ }^{4}$. Most families of children with childhood developmental issues receive services in the public system. The waiting time from the speculation of a child having an ASD tendency to receiving assessment and diagnosis and then to receiving intensive services can take 13 to 19.6 months ${ }^{5}$. Other countries have diagnostic delays ranging from 7 to 30.6 months $s^{6,7}$.

During the waiting period, caregivers face stressful demands to meet the needs of the children and their own needs and have minimal support ${ }^{8}$. Previous studies have shown that parent-mediated programmes play important roles in improving developmental outcomes for children with ASD and developmental delays and caregivers' stress can be alleviated by encouraging caregivers to reach out for professional

and informal support ${ }^{9-11}$. An empirically driven, parent-mediated programme is much needed in HK. Such a programme could address the needs of an increasing number of caregivers of children diagnosed with or suspected of having ASD or developmental delays. This programme could provide a systematic platform for caregivers to give peer support to each other.

In consultation with experts and caregivers' associations from all WHO regions ${ }^{12}$ and findings from several meta-analyses ${ }^{13-15}$, the WHO Caregiver Skills Training (WHO-CST) programme was developed as a task-sharing parent-mediated programme. The WHO-CST programme is part of the initiative of the Mental Health Gap Action programme (mhGAP) for scaling up care for mental, neurological, and substance-use disorders in low- and middle-income countries. The main aim of the WHO-CST is to train non specialist providers, such as social workers, teachers, and community leaders, to educate caregivers of children aged 2 to 9 years old with suspected ASD or development delays. The trained non specialists have essential skills and knowledge to teach caregivers to engage their child in communication and play, promote adaptive behaviours and learning, and reduce challenging behavior ${ }^{16}$. The programme utilizes three individual home visits and nine group sessions.

There are a limited number of specialists in child and adolescent psychopathology in HK. With guidance from the WHO and Autism Speaks, we adapted and have prepiloted the WHO-CST since mid-2018. The main purpose of the programme is to introduce and sustain an evidence-based, low-cost, task-sharing programme for caregivers of children with potential ASD or developmental delays. The programme aims to address the long waiting period for formal diagnosis and treatment in HK.

\section{Method}

The adopted adaptation procedures and research methodology followed recommendations by WHO in Adaptation and Implementation Guide of the Parent Skills Training Programme for Families with Children with Developmental Disorders and Delays ${ }^{17}$ and WHO Parent Skills Training Programme for Caregivers of Children with Developmental Disorders: Monitoring and Evaluation Framework ${ }^{18}$, respectively. The overall study was a three-stage, mixed methods, implementation evaluation approach including (1) formative qualitative research that focused on adaptation of the programme to the local context; (2) a prepilot field test evaluation that examined the programme feasibility and acceptability; and (3) a pilot evaluation that 
assessed programme feasibility and acceptability on a larger scale, using a more rigorous research methodology to enhance the sustainability of the adapted programme. The Human Research Ethics Committee of Hong Kong University (HKU) approved the study (EA1901033). All data collection was performed in accordance with HKU guidelines and regulations. All participants provided written informed consent to (1) participate in the WHO-CST-HK and understood that they could withdraw anytime; (2) join the three home visits that were video-taped and with the presence of their children and they consented on behalf of their children; (3) to participate in the online evaluation as part of the study; and (4) understood that the data collected during the study without identifiable information or image will be destroyed five years after the first publication of the study. This paper reports the findings of the first two stages of the three-stage monitoring and evaluation framework proposed by the $\mathrm{WHO}$.

\section{Stage One - Formative Qualitative Design}

Four types of participants were involved in stage one, representing the key stakeholders for adapting, delivering, and receiving the WHO-CST programme in HK (WHO-CST-HK). They included (1) the core local adaptation team as part of the adaptation advisory group; (2) the Master Trainees (MTs) who received training from the $\mathrm{WHO}$ and were responsible for training facilitators to deliver the programme; (3) the facilitators who delivered the WHO-CST-HK to caregivers at the pilot stage; and (4) the caregivers. The comments from the participating stakeholders were collected to assess whether the programme content was comprehensible, culturally acceptable, and relevant for local families. Feedback from participants in the prepilot WHO-CST-HK was collected to maximize accessibility, feasibility, and acceptability, and reduce foreseeable barriers to participation in the WHO-CST-HK. We conducted two adaptation meetings with stakeholders and focus groups with caregivers during the first two evaluation stages.

\section{Recruitment and Sampling}

At the inception of the programme in May 2018, the research team visited government officials, heads of child and adolescent health services, and directors and management committees of nongovernmental Organisations (NGOs). The team aimed to introduce the WHO-CST, its adaptation process, and to conduct a preliminary situational analysis of how best to embed the WHO-CST into existing services for families of children with ASD or developmental delays in HK.

In October 2018, email invitations to participate in the first adaptation meeting were sent to the Food and Health Bureau, Education Bureau, Department of Health, Hospital Authority, and NGOs to identify their representatives as the core local adaptation team. This core team, together with MTs, and facilitators of the WHO-CST-HK gave advice throughout the adaptation process in the local context. In January 2019, we invited the same group of stakeholders to the second adaptation meeting and updated them on the progress of the adaptation and to collect further comments. Nongovernmental Organisations were also invited to identify eligible caregivers through their social networks to participate in the need assessment qualitative focus group interviews. All interested parties were contacted by the research team and briefed on the interviews, and research participation consents were sought. 


\section{Data Collection and Analysis}

The visits to the stakeholders were informal and, hence, the documentation was not included in the data analysis. The first adaptation meeting discussed (1) sustainability; (2) training and supervision of MTs and facilitators; (3) adaptation and implementation plan; (4) data compiling and reporting; and (5) expected outcomes and deliverables. In the second adaptation meeting, we collected the stakeholders' feedback on the linguistic, cultural, contextual, and methodological considerations for the adapted WHOCST materials. Both meetings were conducted in the university setting and were videotaped, and discussions were transcribed for data analysis.

We conducted in-depth interviews through semi-structured focus groups with caregivers with experience of participating in any parent-mediated programmes in HK. These interviews explored (1) the participants' needs and preferences concerning the parenting programme; (2) possible barriers to attending the programme and potential opportunities to link the programme to other community services or support group activities; and (3) feasibility and acceptability of the WHO-CST content and strategies.

Caregivers first completed a short questionnaire on their sociodemographic characteristics, previous experiences of participating in parent training programmes, and their children's clinical characteristics. They were then invited to answer the following questions:

(1) Could you share your experiences for recruitment and preferred settings for implementation?

(2) Instead of using medical diagnoses as inclusion criteria for our programme, we are planning to use the Ten Questions Screen to screen and identify eligible caregivers, which may increase the chance for caregivers to participate in the CST programme. What do you think?

(3) Caregivers attending the programme will be asked to practice strategies with role play and then to use these strategies at home with their own children. Will there be challenges to caregivers like yourself?

(4) Caregivers participating in the CST programme will be asked to attend nine weekly group sessions and three home visits. Do you foresee any barriers with that?

(5) What do you expect the attributes of competent master trainers and facilitators to be?

(6) Do you have any suggestions for what to be aware of in implementing the CST programme?

Field notes were taken to capture nonverbal cues during the focus group interviews, which lasted between 45 to 70 minutes. All the audio recorded interviews were transcribed verbatim in Cantonese by the research team. Conventional content analysis was used to detect manifest and latent meaning from the data ${ }^{19,20}$. Identified codes with a core meaning for the participants' perceptions were extracted from the transcripts and entered into a qualitative software programme (i.e., NVivo) for subsequent analysis. Rigour was achieved through a process of reflexivity, documenting all analytic decisions, and leaving an audit trail. To ensure plausibility, two independent researchers in the team scrutinized the codes and 
subcategorizations from all the translated transcripts until the reliability reached at least $90 \%$. Regular meetings of the full research team were held to resolve any differences in coding, to refine the analysis, and to determine the final categories. The findings of the qualitative interviews were extracted for modifications of the WHO-CST treatment manual as recommended by the WHO.

\section{Stage 2 - Prepilot Field Test of the Adapted Materials}

This research stage aimed to field test the adapted materials in a small-scale exercise, with qualitative and quantitative information gathered on the acceptability, comprehensibility, and relevance of the materials to the target population. We adopted a single arm prepost group study methodology, and the field tests took place at the premises of the NGOs providing ongoing social and childcare services to families of children diagnosed with ASD and other developmental delays.

Taking into account the number and availability of MTs and facilities, it was proposed to recruit 36 caregiver-child dyads if they fulfilled specific inclusion criteria. The inclusion criteria for caregivers were that they should be at least 18 years or above, responsible for the role of parenting the child, living with the target child, able to communicate in Cantonese, planning to stay in HK for at least six months, and accessible by phone. The inclusion criteria for children were that they should be between 2 to 9 years old and screened positive on any of the modified Ten Questions Screen questionnaire items for developmental delay and disorders ${ }^{21}$. Caregivers were excluded if they fulfilled the following criterion: a physical or mental condition that required hospitalization or frequent outpatient visits (over twice a month). Children were excluded if they fulfilled one of the following criteria: deafness or blindness in the child or comorbid physical and mental conditions requiring the child to be hospitalized.

\section{Recruitment}

Convenient sampling was adopted. Email invitations and flyers were sent to collaborating NGOs. Caregivers who indicated their interest in participating were screened with a brief screening tool by the research team. The chosen measures for the quantitative evaluation were suggested as the core measures for evaluation by the $\mathrm{WHO}^{18}$. All measures were back-translated from English to traditional Chinese by bilingual research members who were at doctorate level. Both caregivers and facilitators who had participated in the prepilot WHO-CST HK groups were invited to complete the prepost questionnaires.

\section{Measures}

Caregivers were asked to complete 23 sets of questionnaires throughout their participation. The 23 sets of questionnaires included one screening tool; one preintervention and one postintervention questionnaire, which asked for demographic and service history information during baseline measurement; eight caregivers' feedback on CST sessions; three knowledge and skills tests; three quality of life tests; two caregiver diaries; and three caregivers' feedback on home visits. Each group of questionnaires was implemented by two MTs. Each MT was responsible for nine surveys for each participating caregiver pertaining to their overall performance during each session, and nine 
questionnaires evaluating the conduct of each session. Similarly, the delivery of the intervention was monitored using video records of demonstrations in the sessions and home visits. The measurements included were as follows:

(a) Caregiver Knowledge and Skills. The Caregiver Knowledge and Skills Test was used to assess the knowledge and skills of the caregivers related to the WHO-CST programme materials. Caregivers were invited to rate 24 statements on a 5 -point Likert scale ranging from 1 (I strongly disagree) to 5 (I strongly agree). Statements, for example, included "My child has more opportunities to learn when we are focusing attention on the same toy or activity". Caregivers were asked to indicate their confidence level (13 statements) on a 5-point Likert scale ranging from 1 (not confident) to 5 (very confident), with statements such as "I feel confident in using pictures to help my child follow a routine". Caregivers were also asked to complete three scenario-based short answer questions. This instrument was used during the first home visit (before the WHO-CST programme), at postsession 4 (mid WHO-CST programme), and during the third home visit (after the WHO-CST programme).

(b) Quality of Life Test. The general health questionnaire (GHQ-12) was used for quality of life assessment to understand the state of health of the caregivers throughout the intervention period. Caregivers were invited to rate 12 statements on a 4-point Likert scale ranging from 0 (never) to 3 (often), for example, "I am feeling unhappy and depressed". This instrument was used during the first home visit (before the WHO-CST programme), at postsession 4 (mid WHO-CST programme), and during the third home visit (after the WHO-CST programme).

(c) Caregiver Experience of Using Intervention Strategies. The Caregiver Diary was used to evaluate the caregivers' experiences with the intervention strategies they learned during the WHO-CST programme. Their diaries recorded their level of confidence, comfort in using the strategies, and how difficult, effortful, and natural the caregiver perceived the strategies to be ${ }^{22}$. Each caregiver was invited to complete the diary in accordance with his or her experience. This instrument was used at postsession 4 (mid WHO-CST programme) and during the third home visit (after the WHO-CST programme).

(d) Caregiver Involvement. The Observer-Rated Involvement of Caregiver During Training Session was completed by one MT or one researcher (who played the role of observer) to assess their beliefs regarding the caregiver's adoption and implementation of the WHO-CST programme strategies ${ }^{22}$.

(e) Attendance Tracking. Caregivers' attendance at the group sessions and home visits was closely monitored and documented by the MTs.

(f) Postsession Feedback From Observers, Master Trainees, and Caregivers. The postsession feedback form - observer, the postsession feedback form - MT, and the postsession feedback form - caregiver were completed by the observer (e.g., the researcher), each MT, and each caregiver, respectively. These forms enabled these participants to share their opinions about the quality, usefulness, relevance, and acceptability of each WHO-CST group session. 
(g) Home Visit Feedback from Caregivers. The Home Visit Caregiver Feedback Form was completed by the caregivers during each home visit for evaluating the usefulness and acceptability of home visits.

\section{The World Health Organisation Caregivers Skills Training Intervention}

The CST was delivered in five NGOs in the community settings by a pair of MTs where at least one of them had passed the basic fidelity requirement of having one play video with over $75 \%$ accuracy accredited by the WHO trainers. The interventions included three home visits and nine group sessions. A set of PowerPoint slides was developed so that all MTs could have a standardized version and only minimal changes were encouraged. The potential caregivers were initially recruited by the MTs in the three NGOs and then screened by the research team for eligibility. Family members of the core caregivers were also encouraged to join the CST. All sessions were videotaped by tablets with tripods, with consent from the caregivers. All videos were then transferred and stored in a computer and server in the university with limited access only by three members of the research team.

\section{Data Collection}

Prepost Intervention Quantitative Data. An online survey tool was used to collect data from different parties, including MTs, caregivers, and researchers using hand-held devices, such as smartphones or tablets. All data collected were kept confidential and were for research purposes only. Each participant was assigned a unique identification (ID) code. The ID code was used instead of the name of participants to maintain anonymity and, thus, the identity of participants could not be disclosed to unauthorized people. The ID codes with names were stored separately from the collected data. Data with identifiable information were kept in a locked cabinet, which could only be accessed by the research team.

Prepost Intervention Qualitative Interview. Focus group interviews were conducted immediately before and after programme implementation. The interviews aimed to explore the trainers' experience delivering the intervention and supporting facilitators as they implemented the programme with the participants. The focus groups collected facilitators' experiences delivering the home and group-based sessions with the caregivers and their children, caregivers' lived experience in the programme, their process of learning about and engaging in the strategies in the programme, and any suggestions to further adapt and improve the intervention.

Process Evaluation. The prepilot groups were delivered by a pair of MTs in each group. One MT was mainly responsible for the delivery of the CST to caregivers, and the other MT was responsible as an assistant and observer to record questions or comments raised by the caregivers that could indicate a lack of comprehensibility, acceptability, or relevance. Feedback from the observers were collected throughout the process to note any issues, such as instructional pacing, time allotted for activities, and the physical teaching environment.

\section{Data Analysis}


All focus group interviews were audiotaped and transcribed. Pattern coding was carried out to summarize major themes in the focus group interviews ${ }^{23}$. Focus group interviews were coded by two coders. The first coder was a trained research assistant majoring in psychology and the second coder was a trained research assistant attaining a master's degree. The coding scheme was developed by the first coder after she had read all the transcripts twice. The second coder coded the transcripts by using the coding scheme developed by the first coder. The interrater reliabilities for the focus groups among social workers were .96 and .97 . For quantitative data, descriptive statistic and repeated measure analyses was conducted to measure prepost changes in mental health and confidence in caregiving. A paired-sample ttest was used to measure the prepost changes in caregivers' knowledge attainment throughout the intervention.

\section{Results}

\section{Stage One - Formative Qualitative Study}

\section{Participants}

Consultation Meetings with Stakeholders. A total of 34 stakeholders including the representatives of government bodies, NGOs, and caregiver attended the meeting. Two representatives from WHO and Autism Speaks, and all MTs also attended. The following section reports their perspectives on the WHOCST in the first adaptation meeting.

Sustainability and the Local Context. The stakeholders agreed that sustainability should be supported by building the programme into existing services in the health care and social service systems, such as a community-based rehabilitation programme, a community-based child health developmental service or other types of health services. There are 19 Parent Resource Centres (PRCs) providing community support for the parents and caregivers of persons with disabilities throughout the 18 districts in HK. It was suggested that parents and caregivers could participate in the WHO-CST-HK delivered by staff who run the PRCs and caregivers could also exchange lived experience and establish mutual support.

Opportunities. There are a growing number of health care and social services for families of children with ASD or developmental delays or disorders. Many stakeholders were, therefore, supportive that implementing WHO-CST-HK is essential as an intervention for the entire community. The implementation should be "as inclusive as possible" and should address the needs of as many families as possible:

(For) child and adolescent psychiatric services, we have a severely long waiting time, hence there is an extreme demand for services.

Most parents are misinformed or uninformed about what is really happening in HK. Many parents are deprived of information... Let's find a way to reach those who are in need. The very purpose of the (WHOCST) programme is to discover how to reach out to people at a low cost, make it sustainable, and make it effective...so (let's) make it inclusive. 
Many parents in HK are in need. No matter whether their children are diagnosed with special needs or not. They need these parenting and play skills.

In addition to fulfilling familial needs, one stakeholder highlighted that teachers in kindergarten and primary schools also need to learn the skills offered by the WHO-CST-HK:

To a certain extent, teachers also need these skills, as they may not be intensively trained in (the) application of behavioural management techniques or communication techniques with children.

Home visits were identified as a barrier for implementing the WHO-CST-HK. It was mentioned that some families may be over-concerned about confidentiality and reluctant to, or refuse to, invite "strangers" to visit their homes for evaluation and the provision of services. However, some stakeholders reflected that in their experiences of conducting parent training programmes, home visits are important to build up initial engagement between the interventionists and the caregivers. Families welcome home visits if the purpose has been explicitly stated at the outset. It was suggested that interventionists could evaluate whether the caregivers could apply the techniques learned during sessions in real-life situations:

We conduct the home visit again to know what they have learned from the group and how they apply it in their home settings....So we understand that home visits are very important to understand the family and their needs. Some of my colleagues (who) conducted home visits will know, in a real living environment, what kinds of strategies they can apply or cannot apply.

Challenges. Although the WHO-CST-HK was generally well-accepted by most stakeholders, some challenges regarding its sustainability were identified. One challenge was related to the perceptions of interventionists on programme implementation in the primary health care settings. One stakeholder pointed out that some of the potential interventionists in the health care settings are nurses who may not be ready to shift their delivery of care from tertiary sectors to primary sectors:

The health care services in here have been skewed in secondary and tertiary care...the Hospital Authority is now shifting the services to a more community-based level, (focusing more) on maintenance care and preventive measures...It was a great challenge, (since) not all psychiatric nurses are ready to equip (themselves) to deliver services to primary health care. It is a new chapter for us to reach out and to collaborate with community partners. It is another set of competencies that they need to develop and to communicate.

Training and Supervision of Master Trainers and Facilitators. It was suggested that MTs could be general or specific health care providers, such as pediatricians, psychologists, mental health nurses or counsellors, while the facilitators could be community health workers or peer caregivers. Contrasting views were revealed among the stakeholders regarding the need for professional qualifications for the interventionists (i.e., either master trainers or facilitators). Some stakeholders supported the idea that peer caregivers could be the interventionists (i.e., facilitators), given that they are more willing to share their 
caregiving experiences, are committed to providing services to their peers, and willing to build up social connections within their peer groups:

We welcome parents or nonprofessionals as facilitators. Many years before we ran our training programmes in parent resource centres, and we trained a group of parents. They went through the difficulties and they want to be the volunteers to partner with other parents of children who are newly diagnosed with ASD. So, we provide some training for them and they became the coaches for other parents.... It's very meaningful as many years afterwards, these parents still keep contact with the coach, such as "yum cha", and they still support each other in the community in the long run.

Parents could also be the facilitators... Peer parents regularly come to our centres and make some "care calls" to other parents. We also think that facilitators who are the parents of children from different age groups are good because ASD is a lifelong condition. At different life stages, parents encounter different challenges, and they could share their experiences of what they have gone through, so that parents could get some mutual support.

Nevertheless, one stakeholder reflected that some parents in HK prefer professionals to lead the training programmes. She also noted that from her hosting institution experience, which had provided training to parents in $\mathrm{HK}$, it was difficult to engage them due to their busy working schedule:

In parents' minds, they trust professionals. If the facilitators are not professionals, they may not trust the programme, (for example), ....it is difficult to engage caregivers for sustainable training due to their family and work engagements.

Some stakeholders suggested the need for ongoing training and supervision after the training of MTs offered by WHO and Autism Speaks. Various strategies were suggested based on the stakeholders' experiences of organizing parent training programmes, such as reviewing videotaped sessions and online training:

Ongoing training and sharing among our staff are very important because during the process when we hold different groups, we may come across different types of difficulties or parents'issues. (Hence) we continue with in-service training; we videotape some sessions when running groups. New staff and programme facilitators can watch the videos to learn to facilitate the skills that are more appropriate for our parents.

We may have difficulty in officially releasing staff members to attend the five-day training. It may help if the training can be delivered in another kind of format, like e-training or online training.

Adaptation and Implementation Plan. In the planning meeting, several stakeholders raised concerns regarding the need to limit the number of group sessions (e.g., from nine weekly sessions to six weekly sessions) and to shorten the duration of each group session (e.g., from 2 hours to 1 hour) due to the caregivers' busy schedules. A few stakeholders, in particular, parents' representatives supported following 
the original version of the WHO-CST programme, rather than bringing in preconceived ideas about the programme (e.g., long schedule) without trying out the first round of actual implementation.

Let us not think about efficiency version or whatever... let's just do the full version because that's what it is basically designed for... Adaptation, to me, is to make the programme more efficient, try to connect with people more efficiently and meaningfully. Other than that, we should not make it more compromised. Let us find out and have it implemented in full and see...

Just go straight away, to see how it (the WHO-CST programme) implements with parents as it also depends on the facilitators, how they (parents) interpret and localize it into daily life situations. Adaptation and translation are an ongoing process, with feedback not just from the parents, but also from the facilitators and all stakeholders.

One of the important aspects of the WHO-CST programme is to collect and analyze the data to examine the feasibility and acceptability of the locally adapted programme. These data can also provide core information to guide implementation and the possibility of scaling up the WHO-CST to an international level. However, there were concerns related to the need for ongoing research among service providers in the future. One stakeholder found that it could be a challenge for social workers and other health care providers to be involved in a research study as they perceived that research is not related to service provision. Hence, frequent communication is required to allow staff to understand the rationale for the research:

Sometimes it is difficult for us to have an randomized controlled trial (RCT) study because most of the social workers and frontline workers do the frontline work with the parents only. But for the research study we must commit to lots of things, some administrative work, and some preparatory work...not really related to direct service. So, we must communicate with our staff and colleagues a lot first... we must educate our staff that it is necessary to do research. Sometimes we may forget about the study as we focus on the service.

A few stakeholders raised concerns regarding the difficulty of recruiting eligible and adequate numbers of families to participate in the pilot stage, which is a randomized controlled trial study. One stakeholder, however, shared her experience of collaborating with a university institution to conduct a trial study of a parent-mediated programme and stated that recruiting subjects was not that difficult if the study was carefully monitored and explained to the caregivers in detail. The parents would, in those circumstances, be aware of the importance of commitment throughout the study:

Ongoing monitoring is very important... in the process it is also important to let our target parents know what we are doing because sometimes it is easy for them to drop out. Sometimes their child is sick, or other things. They may have to withdraw, or they may have to skip some sessions. So, at the start, we tell the parents what we are doing and there is research behind it... think we must let the parents know the importance of commitment. 
The programme was regarded as an essential intervention for the local community in response to the rising trend of children with ASD or developmental delays or disorders. Other favourable factors supporting the sustainability of the programme include the availability of venues to support the group sessions and the acceptability of conducting home visits among HK Chinese families. Some foreseeable challenges were revealed during the pilot planning meeting, such as the difficulty of promoting the programme because of the variety of parent training programmes already available.

Review of Draft World Health Organisation Caregiver Skills Training Materials. From April to July 2019, the research team, the MTs, and a few invited individuals, including caregivers, reviewed all backtranslated WHO-CST materials and drafted the localized version. The back translation was completed by two trilingual undergraduate students majoring in psychology and counselling who had a strong interest in ASD and who were supervised by the research team. An official written language instead of a dialect version was used so that the materials could be shared with other Chinese societies. In the review, cultural differences, contextual and methodological considerations, and issues of feasibility and acceptability were addressed, such as whether use of particular wording was acceptable to the local community. Most of the content remained consistent with the original version and only a few key terms were debated. The terms "routine", "show and say" or "show and tell", and "set-up environment" were adapted to the local language. The illustrations of culturally diverse characters and settings remained unchanged. Character names were translated but not altered to local names. Only minimal changes were made to the translated version as suggested by the $\mathrm{WHO}^{17}$.

\section{Need Assessment Focus Group Interviews of Caregivers of Children with Autism Spectrum Disorder. A} total of 15 caregivers (three Nepalese, twelve Chinese), who were mothers, participated in the focus group interviews, one in group 1, two in group 2 and three in group 3, 4, 5, and 6, from 3 to 22 December 2018. All the caregivers identified themselves as their child's primary caregiver. Among the caregivers (age $M=38.1, S D=2.2$, range: 34-41), one was a working mother (part-time) and the rest were housewives. Most caregivers $(n=14)$ had lived with the target child since birth and belonged to nuclear families (see Table 1). They were asked for their view on the acceptability and feasibility of the programme.

All had participated in various types of parent training programmes, such as the Preschool Autism Communication Trial (PACT) ( $n=2)$; Happy Parenting $(n=2)$; Louis Programme $(n=2)$; The Hanen Programme $(n=1)$; The SCERTS ${ }^{\circledR}$ Model $(n=1)$; Applied Behavioural Analysis $(n=1)$ and a mindfulness programme $(n=1)$.

All participants in the focus group interviews supported the implementation of the WHO-CST programme in HK. Since the majority $(n=12)$ had attended various types of parent training programmes, they perceived the WHO-CST programme as "another choice for parents" and "like other supporting programmes organized by NGOs". One mother, who had participated in the Preschool Autism Communication Trial (PACT), mindfulness course, and Happy Parenting programme, expressed her views as follow: 
Parents often need to make lots of effort, but for those who have not received any training, they will not know the cues for helping their child, so that is why they need to attend the course and learn.

Another mother, who had participated in the Social Communication, Emotional Regulation, Transactional Support (SCERTS) model course, shared her views:

If (we) could search for other courses, they will usually introduce what autism really means to us. It is just a sharing only; it will not be as practical as you just mentioned.

Nepalese mothers emphasized the need for WHO-CST because of the language barriers of attending other training programmes, which were all delivered in Chinese:

We learned the techniques from teachers at schools in Chinese...they provide translators to us. But the practical part is in Chinese and that is the major problem for us. At home we used English and my child may not respond to that. So, we messed up everything.

Apart from showing their appreciation and support towards the WHO-CST programme, the participants shared their perspectives on the implementation process of the programme, covering the following areas: (1) directions of implementation and sustainability; (2) inclusion and exclusion criterion for target participants; (3) screening and recruitment procedures; (4) programme content; (5) attributes of MTs and facilitators, and (6) assessment method for impact measurement - videotaping. Furthermore, several suggestions for improving the acceptability and sustainability of the programme were given by these caregivers.

(1) Directions of implementation and sustainability

Be specific and practical. Many participants $(n=12)$ highlighted the importance of establishing clear goals and applicability to real-life practice at the beginning of promoting the WHO-CST programme or scaling up to the territory level. These participants shared their experiences of attending multiple parent training programmes in their lifetime, but finally they found these programmes were too theoretical and could not help their child when applying their skills in real situations. Caregivers are looking for programmes specifically addressing their needs and challenges in caring for their child:

The goal (of the WHO-CST programme) must be made much clearer, what target participants are looking for, because sometimes I found after I participated in a programme, oh (I) joined the wrong course, a wrong one.

The useful stuff I will take it, but not just theoretical inputs, because after you listen to them (note: skills learned from the training programmes), during that moment you may understand, but as time goes by, you would forget. It'd great if that moment it is useful.

One NGO, (it costs) one hundred dollars only (note: course fee), but I still don't want to join, as it's not attractive and has no practical use... it's better than nothing, but if (those programmes) are more target- 
orientated for each individual child's situation that'd be more attractive.

Be universal. Four participants were thinking of whether the WHO-CST could be a universal parenting programme that could serve the families who are in need. One mother, who had been living in Canada since being a teenager, had recently moved to HK. She found that many families in HK with a child with ASD were not yet prepared to receive services, while those who were ready to commit to the WHO-CST (e.g., complete the group sessions and home visits), could access other resources and may not be the ones who "really need the service":

ASD families have ASD characteristics, right at the beginning you need us to commit to so many tasks (note: home visits, video recordings). I believe that you just simply scared people away. Then that means you would have only those families who are opened-up, and even without your programme they could still access services, they are able to search for resources that could support them.

Highlight the uniqueness of the WHO-CST in Hong Kong. During the interviews, almost half of the participants $(n=7)$ asked about the uniqueness of the WHO-CST programme when compared to the other parent-mediated programmes in HK. Those who had joined other programmes before asked, "why do we need an additional one?" Some caregivers mentioned that for improving knowledge, they could search for information from the internet; for self-care, they could join mindfulness courses and other relaxation exercises; for behavioural skills training, they could also join PACT, Positive Parenting Programme (Triple P) or other training workshops.

Every year the school provides similar stuff (note: programme or course) to us, then a single education talk will be fine, it's just similar, so why attend? Even (we need to) attend nine sessions, so please don't bother (me), so some parents may think in this way. Teaching parenting skills... in fact, hey they're just all the same.

I do not know why the WHO needs to organize this programme? There are so many skills training programmes, and there is so much (information) online, up to the point that we cannot digest so much. If (you teach me) ten skills and I could use one to two skills at home, that could save my life, or relieve me. I think that is more important than that three-month WHO programme.

(2) Inclusion and exclusion criterion for target participants

Most participants $(n=10)$ agreed that the child's age range of 2 to 9 as one of the eligibility criteria for joining the WHO-CST programme was acceptable.

Yes, parents of children in different age groups need these supporting programmes. These services should not be discontinued when the child is at primary school.

At the beginning (when the child) is aged 2 years, (he/she) would be alert to what the adults are doing, so it's better to start (the programme) at the age of two. The golden period would be on or before aged 8. That's why all the resources are cut at age 8 for the primary schools and you are required to seek 
(resources) yourself. They are thinking everything is fixed already. There's no more about (the child) that can be changed, nothing can be changed. So for me, if the training could last till aged 9 it's better.

(3) Screening and recruitment procedures

From caregivers' perspectives, whether they are eligible to participate in parent training programmes is due to "chance", while how to increase the reach of the WHO-CST programme is another area of concern among the participants $(n=5)$. One mother recalled her difficult experience of searching for appropriate training courses and she finally realized that her perception of "challenging the accuracy of the screening test" might be the reason behind this difficulty:

Why could I not find these courses at the very beginning? ... Oh! Indeed, when we (note: participant and her husband) knew (the results), I think it was not that case, so we didn't do anything, my husband and I thought it was impossible, we thought he does not have developmental delay, the assessment had problems...

Three participants proposed that, in addition to promoting the WHO-CST programme in NGOs, maternal child health clinics (MCHCs) under the Department of Health and kindergartens could be sites for recruiting eligible caregivers. Most caregivers and their children need to receive services from MCHCs, and it is mandatory for the child to attend kindergarten.

I think in MCHCs we need to have a pamphlet (about the WHO-CST programme) that means to alert the parents, so if you encounter your child's situation, you could search for these programmes to offer help, but it is unnecessary to wait for the services provided by Child Assessment Centre, which means it would be a long waiting journey.

To make the kindergartens better aware (of the child's problem), if some teachers have such alertness, and (they know) the child has such problems, they could tell the (parents) there is a programme (called WHO-CST programme) and suggest they participate in that, which could save time and resources.

Support the use of the WHO Ten Questions Screen to identify eligible participants. Use of the Ten Question Screen, a 10-item questionnaire as a screening tool, was welcomed by the majority of participants $(n=11)$. By using this screening tool, the participants perceived that the health and social service providers trusted the parental reports of the child's developmental problems, and it increased the chance of participating in the WHO-CST programme. In view of the lack of resources during medical consultations in MCHCs or CACs, some caregivers would rather exaggerate how difficult the child's behaviour was at home in order to be diagnosed and offered appropriate resources. One mother described such experiences as traumatizing. If the Ten Question Screen could be used, caregivers perceived the tool to be user-friendly and the WHO-CST would not be limited to those families where their child had received the diagnosis. Those awaiting diagnosis by health care professionals could also receive the service. 
I am so excited about what you've just mentioned (note: about the Ten Question Screen). I'd say please do not rely on those reports. Those reports are based on what the parents told (to the doctors) during the interview. Often parents would share what they perceived their child's problems to be. Because of fighting for resources, (many parents) would rather seek for the reports, so if (you) want to serve the parents, the principle is to ask less. It's not necessary to obtain so many child reports.

(4) Programme content

Programme setting, timeslots, and session number. Some participants $(n=3)$ suggested that the venue for the group sessions should be convenient for them (e.g., near their home) and the preferable timeslots would be during weekday mornings (i.e., during the child's school days). Nevertheless, half of the participants $(n=6)$ found that the group sessions arranged on a weekly basis would be "too rushed" or "too packed" for the caregivers. If the WHO-CST programme is a multicomponent programme, it was recommended to provide time for the caregivers to understand the materials and to practice in real-life situations:

Too rushed. I have not understood most of the materials and then the next session, the programme (sessions) are too packed, because every week we need to attend, (we have) to understand, to digest, and then to use them. It is very demanding.

Because for my child, it is not like an injection, it's not like after the nine sessions that suddenly (my child) would know everything all in a sudden, it's not like that, it takes time, or during practice if I encounter difficulties, l'd not know how to solve them...

One mother proposed that the group sessions of the WHO-CST programme could be arranged every other week:

The benefit (of arranging the programme) on a bi-weekly basis is it will not be too difficult, for travelling back and forth...otherwise I won't have my own time, or time to rest. (I) won't have time for the housework...

Home visits. Including home visits as one of the intervention strategies of the WHO-CST programme was actively discussed during the focus group interviews with contrasting views. For those participants who supported home visits, they perceived it as an opportunity for building up connections and trust in one-toone relationships. More importantly, the interventionists (i.e., MTs and facilitators) could better understand the difficulties that the caregivers are encountering and offer specific intervention strategies:

You really must visit to his or her home, possibly (you) may realize lots of issues, and (the interventionist) could directly point out the issue, and demonstrate to you at once, (for example), how difficult it could be to help the child to wear a pair of shorts, even though for helping the interventionists, I think they could understand. 
It'd better to have some "tailor-made" time for us, that means (we could) raise questions, such as how to manage our child's problems, for the home visits, if some professionals could provide one-to-one service, that'd be appropriate. Because there are many training programmes that could serve general situations, but they're not specific enough...

You come and understand what exactly the problems are currently facing each individual family, and then the trainer could know how to train the entire family as the ASD problem could be associated with the entire family unit.

One mother from an extended family suggested that interventionists may play a significant role in teaching other family members in managing the child's problem.

Face-to-face (interviews) could allow the interventionist to get in touch with the entire family, teach the grandparents, or teach the father or the brother, so (home visits) are good, and the interventionist could observe the entire home environment.

Some participants hesitating about the home visits think that their living areas are too small and that may not be favourable for home visits. If the caregiver is living with other extended family members, such as grandparents, home visits may not be acceptable among these families.

You know the living space is small in HK, that means I may not (allow home visits), and for those living with grandparents, they may even refuse the home visits, and even three visits seem too much.

One mother raised a concern that the Chinese term for home visits symbolized that the child may not behave well at school:

With the term "home visit" I could feel pressurized, as in HK, the only issue when you need a home visit is because your child misbehaves at school. You'd immediately think of something bad (about your child).

(5) Qualities of master trainers and facilitators

Professionalism of MTs and facilitators is another concern raised by some participants $(n=5)$. From caregivers' perspectives, those who had attended various types of training programmes before expected MTs and facilitators to be skillful, knowledgeable, and down-to-earth. These caregivers did not want their child to be "tested" or "experimented on":

Maybe for those so-called professionals who just received training from a lecture, (they) may not have much experience in managing a child, because studying and conducting real-life practice is different. I am expecting that at least they have experience in caring for these children, know how to play with them, and know how to communicate with them.

For those who just recently graduated and are just treating your child for the sake of doing an experiment, then please don't. It's not that I don't want to spend the time. We don't have much time to be spent (in this 
way). And I even don't have time to tackle our child's problem, so how come I would have time to spend for those so-called professionals.

(6) Assessment method for impact measurement - videotaping

Videotaping the child's interaction with the caregiver as an assessment method of the WHO-CST programme was the issue that raised the most concern during the focus group interviews. Some participants thought that videotaping at home would make them feel uncomfortable because of the issues of privacy and confidentiality. Furthermore, a couple of enquires were raised among the participants, such as "what is the purpose of videotaping?"; "how would the videotapes to be handled?"; and "will the video records be disclosed for publicity, public education, reviewing with other caregivers during group sessions, and/or researchers to do the analysis?".

I am so afraid, oh my god if you really did the video recording, how would you handle the tapes?

Because it's just like taking off all your clothes. Let the others see what's going on. Your home is so messy, or your home is that dirty. Parents do mind this, would have feelings of discomfort.

If video recordings are made I could not be that relaxed, and l'd considered the video records as taboo. There'll be some requirements. (I) don't feel very comfortable with that.

One mother raised another concern about whether videotaping could impact on the effect of the WHOCST programme on caregiver-child interactions, given that the behaviour and emotions of children with ASD can fluctuate significantly.

The child's performance could fluctuate, because even if (the child) is at home, for that moment, whether you could record that situation it's hard to say. You may not really be able to see their improvements, and I think (that improvement) it's just by chance, so I think you may not see the effect.

(7) Suggestions for improvement

At the end of the focus group interviews, the participants proposed several strategies that could improve the feasibility and acceptability of implementing the WHO-CST programme. Some participants suggested conducting a briefing session for all the eligible participants of the WHO-CST programme before the first round of home visits. This briefing session could particularly address the need for home visits and video recording so that caregivers would have a clearer idea of the whole implementation process.

Another noteworthy issue was related to ongoing support after completing the programme. Using followup calls, apps, focus group interviews, and establishing an online platform for the caregivers to share their caregiving experiences were strategies proposed:

After I complete the programme the service would be immediately cut off, so what shall I do? If it isn't, we do need to have back-up, can approach the nurses or social workers. It could be apps, it could be very 
simple, just like these focus group interviews. (We) could have a very informal discussion, these could help the parents a lot...

There is a platform for us to share, if I encounter this problem, if I forgot something, or anything that (I) could do better...indeed I found (this support) is important.

Poor couple relationship is one of the problems that was raised by several participants $(n=3)$. These participants found that their husbands find it difficult to accept that their child has ASD or developmental delays. They suggested that the WHO-CST programme content could also address couples rather than single caregivers.

My husband still doesn't accept (the child as having ASD). It has been three years that he doesn't accept it. The parenting strategies won't be consistent with each other. There are lots of arguments throughout the day. An all-rounded (programme) would be better that serves couples, for one to two sessions that serve couples, it'd be great.

In summary, all the participants had experiences of participating in various types of parent training programmes. Most participants welcomed the WHO-CST-HK and felt it would fill the gap caused by increasing service demand for families of children with ASD, developmental delays, and developmental disorders. Nevertheless, the participants highlighted a few potential enhancements during the programme implementation. For instance, the programme aim needs to be specific, the content needs to be practical and universal, and serve families who are in need. The uniqueness of the WHO-CST programme, when compared to the rest of the existing local training programmes, should be emphasized.

\section{Stage Two - Prepilot Field Test of the Adapted Materials}

\section{Participants}

(I) Pre and post CST programme qualitative evaluation with focus group interviews

Thirty-eight caregivers of children with ASD joined pre-CST programme focus group interviews immediately after they had attended the briefing session at the HKU for joining the WHO-CST-HK between 23 March and 28 May 2019. There were seven participants in group 1, six in group 2, nine in group 3 , three in group 4, four in group 5, six in group 6 , and three in group 7. While some had proceeded to attend the CST prepilot sessions, others had terminated due to scheduling issues or were lost in the follow-up, resulting in 18 caregivers remaining to participate in three post CST programme focus group interviews between 22 July and 20 September 2019. There were five participants in group 1, two in group 2, four in group 3 , three in group 4, and three in group 5. During both interviews, focus group guides developed by the WHO were used to direct the flow of the group discussion. The Chinese version of the guides was adapted by the postdoctoral fellow and circulated and agreed among all the MTs and the principal investigator. All focus group interviews were conducted by researchers of HKU with the assistance of the involved MTs. 


\section{Preintervention focus group}

Through the focus group interviews with caregivers, and the major themes included 1) perceived benefits of the programme, 2) useful elements in the programme, 3) environmental and cultural barriers, and 4) major challenges and proposed solutions.

Prior to joining CST sessions, caregivers expressed hopes for improving communication between themselves and their child, better understanding the needs of their child, and preparing their child for social situations.

So I want to communicate more with (my child), firstly for more communication, and secondly to engage in paired reading more often.

...teach him how he should express himself and how he should act.

Some caregivers were expecting to learn skills that would better fit their own situation.

Usually, after learning skills from other courses, they are hard to implement back at home. Now that you will come to my home, I am hoping that you could tell me how to better train my child in my own setting.

Some caregivers were hoping to understand and manage their child's inappropriate behaviour.

Sometimes he doesn't like to wash his hair, he can throw a big tantrum like crazy. I want to learn something about it.

Many caregivers expressed hopes that home visits would help them in implementing the skills taught during sessions, as well as help them pick and choose the right skill to use. However, many of them came across the barrier of their other family members not supporting their decision to join the programme.

I and my wife hold different opinions on the matter. That's why sometimes you will see me accepting certain situations, but she doesn't. Sometimes I think it is right, but she does not, something about order. We also have an elderly relative at home who may be uncooperative at times.

Many caregivers understood that the older generation may have a different caregiving method and were worried that it might clash with skills taught in CST. In addition, caregivers lacked confidence in carrying out home practice. Caregivers expected the child to be uncooperative during practice and were not confident that they could apply the learned skills.

Even if you asked him, he won't come over.

Most reported expectations were child-orientated, hoping that CST would directly impact on their child.

Postintervention focus group 
During the postprogramme focus group interviews, five prominent themes emerged: 1) overall reduction in challenging situations, 2) improvement of caregiving skills, 3) management of own emotions as a caregiver and taking better care of themselves, 4) understanding and management of child's inappropriate behaviours, and 5) home visits as a helpful element.

After participating in all the CST sessions, caregivers reported observing a significant reduction in challenging situations.

Initially we would fight over something constantly, but later in the programme, the fighting reduced. There is literally no more fighting over anything.

There is no third choice, nothing to choose.

Caregivers learned to offer only two choices, with no third choice for bargaining, and the child must pick between the two. As a result, many felt that they had improved on their caregiving skills. Caregivers also recognized the importance of managing their own emotions, particularly after the last session. They reported placing more focus on themselves, which helped them improve in caregiving skills. Caregivers indicated that CST helped them understand their child's inappropriate behaviour from a different perspective:

Now that I have taken the course, I understand my child's behaviour. I know what methods I could use to help him, now his (behaviour) improved. My angle and stance changed.

All caregivers also agreed that home visits were particularly helpful in enhancing caregiver skills. Many stated that they would like more time per visit, and some wanted to have a copy of their own video for revision and as an example for other family members.

(II) Pre and post CST programme quantitative evaluation with questionnaires

Forty-two caregivers joined six WHO-CST-HK groups in the prepilot. Approximately $80 \%$ of caregivers were female (age $M=37.12, S D=4.16$, range: $30-44$ ), $21.4 \%$ of caregivers were male (age $M=39.44, S D=6.82$, range: $30-49)$. In general, for most of the chosen scales for evaluation, statistically significant differences were not found before and after the programme participation in the caregivers' mental health and confidence in caregiving (see Figures 1 and 2, respectively). Participants found that most of the 12 sessions, and especially the home visits, were relatable, useful, and helpful. Caregivers generally found the materials of session 8: problem solving, and self-care to be the most relatable $(M=4.09, S D=.82)$ and useful $(M=4.13, S D=.80)$.

\section{Discussion}

This paper presents the process of exploring the acceptability, feasibility, and initial impact of implementing the WHO-CST in HK. It is noteworthy that although the WHO-CST was originally developed for low and middle resourced countries, the programme was acceptable. Core elements of CST, such as 
home visits, were highly appreciated by the stakeholders and caregivers because they gave a sense of tailor-made intervention for the caregivers. It was understood that if positive research findings of the WHO-CST-HK could be documented in the pilot stage, it is very likely that the CST can be sustained at least in the social service setting. It was further understood that sustaining the programme could help address the increasing number of people diagnosed with ASD or developmental delays. The programme could help achieve "zero-waiting time" for families of children with suspected special needs, as proposed by the Chief Executive in her Policy Address in $2018{ }^{24}$.

By adhering to the WHO's guidelines for adaptation, monitoring, and evaluation of the CST, we have collected a wide spectrum of in-depth qualitative and quantitative information, which indicates that the adapted CST programme is acceptable in the local context and is ready for a larger-scale field test with a more rigorous research methodology. During the formative qualitative research stage, most of the stakeholders agreed that the CST would become a culturally relevant parent-mediated programme for families of children with special needs in HK. Although a few stakeholders initially worried about the feasibility of including home visits, the evaluation component, and the length of the CST, caregivers in the needs assessment focus groups stated that they were attracted to the programme because it was developed and delivered by reputable Organisations and it seemed that the WHO-CST-HK is distinctive compared to other programmes they have joined.

There are some aspects of the WHO-CST-HK that require minor adjustment or special consideration during the adaptation process. First, MTs reported that course content may not be suitable for older children within the initial age range (2-9) suggested by the WHO. In particular, the experienced practitioners stated that the content on training a child's language skills is not suitable for local caregivers because most participated caregivers had children who have already attained fluency in language production. This phenomenon is probably related to the strong focus on academic success and the importance of language in schooling among all caregivers in HK. The caregivers tend to see mastery in speaking and communication as the prerequisite for academic success. Hence, caregivers seek as much professional help as possible to support their children in acquiring language skills. Therefore, the language section in the CST materials is less discussed in comparison with other sections.

Second, the MTs reported that the fidelity training delivered by WHO and AS in the CST is unique and much needed to enhance the quality of service for caregivers. This fidelity training was important in clarifying their understanding of the course content. However, the process from completing the fidelity assessment to receiving the performance report and accreditation in becoming a qualified MT was too lengthy for this three-year territory-wide project. Unlike other CST sites currently under the implementation and evaluation process, the adaptation of the CST in HK is part of a HK-wide project focus on ASD training in school settings. As a consequence of this focus, the research team has very little flexibility to adjust the timeline of the adaptation, prepilot, and pilot monitoring and evaluation process. Since there is already a territory-wide project, a high number of qualified MTs are needed to meet the requirements of the pledged output for the funder. Given the context, the chief trainees from WHO and AS are responsible for the training and assessment of all research sites of the CST around the world. It is recommended that 
a regional trainee system is much needed to speed up the process of MT and facilitator training for largescale implementation of the CST programme in the future.

Third, some caregivers who had already participated in other parent-mediated programmes in HK expressed their views concerning the length of WHO-CST-HK being delivered weekly. These caregivers argued that the weekly schedule was too rushed and may affect their learning. Unlike other parentmediated programmes in HK, the CST has many practical sessions to consolidate the learning of the caregivers on play and home routines to enhance parent-child interaction. Overall, the pace of the implementation of the CST was accepted and this is reflected in the positive preliminary results of the prepilot stage.

The WHO-CST has created a positive impression for all the stakeholders. First, with experienced professionals involved in the recruitment and implementation process, eligible caregivers were easily identified and recruited to join the prepilot field tests. There is significant demand for empirically driven and well-designed parent-mediated programmes to meet the needs of the increasing number of families with children with ASD or delayed development. Second, the MTs have good professional and clinical backgrounds concerning ASD, which made the delivery of the CST feasible and well-received by the caregivers. Initial quantitative evaluation findings suggest that most of the CST sessions and all home visits were perceived as relatable, useful, and helpful. This may be because the programme placed caregivers with the same MTs who could help foster trust and openness ${ }^{25}$. The application of home visits also tailored personal plans, which could empower caregivers to achieve their goals ${ }^{26}$.

The WHO-CST-HK has covered multiple areas of caregiving, which might have facilitated caregivers to form a cohesive picture of understanding so that they could follow through when dealing with challenging situations. In HK, many caregiver education programmes focus on persuading the participant to improve themselves but ignore skills that could help caregivers identify the source of the problem from other ${ }^{27,28}$ \{Fabrizio, 2015 \#101;Stewart, 2012 \#104\}\{Fabrizio, 2015 \#101;Stewart, 2012 \#104\}\{Fabrizio, 2015 \#101;Stewart, 2012 \#104\}\{Fabrizio, 2015 \#101;Stewart, 2012 \#104\}.\{Fabrizio, 2015 \#101;Stewart, $2012 \# 104$ \} For most sessions, at least one skill taught was found useful by caregivers. With skills such as offering only two choices, caregivers understood that they can improve on their caregiving skills. As a result, they observed a significant reduction in challenging situations on a daily basis. However, as WHOCST is evidence-based and utilizes skills that have been proven beneficial from other caregiving programmes, caregivers may have already heard of or learned certain techniques from other sources, therefore, their knowledge attainment was not significant in this programme.

Caregivers also reported observing the positive impact of WHO-CST in their daily lives. During the focus group interviews, caregivers were able to explain how they used the specific CST skills to respond to their situations. Consistent with the literature, home practice sessions and discussion on caregiver's experience is an effective method for ensuring caregivers understand and use the skills taught ${ }^{29}$. However, previous programmes of a similar nature were usually conducted in a one-on-one format and caregivers in those programmes lacked the opportunity to develop the sense of peer support ${ }^{29,30}$. In CST, through discussing 
caregivers' personal experiences in a group, caregivers and facilitators could work together and generate suggestions, based on CST principles, which resulted in caregivers observing a significant reduction in challenging situations in daily activities such as dining and teeth brushing. As children responded to the improved caregiving skills, there was a reduction in children initiating challenging behaviour.

\section{Conclusion}

Given the heavily reliance on the public system by the caregivers of children with ASD and the lengthy waiting time to receive services, the WHO-CST-HK is regarded as an acceptable and useful parentmediated programme to support families of children with ASD in HK. However, probably due to the prepilot nature and the small sample size of the prepilot evaluation, quantitative findings on the chosen scales have been statistically insignificant when preintervention and postintervention periods are compared. The received qualitative feedback, however, is very positive. The next step for the research team is to conduct a randomized controlled trial with a sufficient sample size to prove that WHO-CST-HK is an evidence-based parent-mediated programme that is helpful in enhancing parent-child interaction, reducing parental stress, and improving children's development. The programme could then be sustained with the support of the WHO, AS, local NGOs, caregivers, MTs, and facilitators in HK.

\section{Declarations}

\section{Acknowledgments}

This work was supported by the Hong Kong Jockey Club Charities Trust and was awarded to Paul W.C. Wong. We would like to thank the participants of the study and the NGOs for their assistance with the participant recruitment. We sincerely wish to thank the research team from the WHO CST for guiding us throughout the research process. We also want to thank Dr Andy Shih, Dr Pamela Dixon, Dr Chiara Servili, Dr Erica Solomone, Dr Laura Paicone and Dr Stephanie Shire for their never-failing support and kind hearts to walk the baby steps with us throughout the years.

Financial disclosure: This work was supported by the Hong Kong Jockey Club Charities Trust.

Author contributions: PW, JL, HKF were responsible for the conception of the research question and PW, HKF, YYL drafted the manuscript. PW, YYL, HKF were responsible for study design, data collection, and data cleaning. PW, YYL, HKF were responsible for data analysis. All authors have approved the final version of the manuscript.

Conflicts of Interest: The author(s) declare no competing interests.

None declared.

\section{Multimedia Appendix}

Nil 


\section{References}

1 Whiteford, H. A. et al. Global burden of disease attributable to mental and substance use disorders: findings from the Global Burden of Disease Study 2010. The Lancet 382, 1575-1586, doi:https://doi.org/10.1016/S0140-6736(13)61611-6 (2013).

2 Census and Statistics Department. Hong Kong Monthly Digest of Statistics. (HKSAR, 2020).

3 Food and Health Bureau. Mental Health Review Report. (Hong Kong, 2018).

$4 \quad$ Food and Health Bureau. Mental Health Review Report. (Hong Kong Special Administrative Region Government, Hong Kong Special Administrative Region, 2017).

$5 \quad$ Hong Kong Special Administrative Region. LCQ14: Child Assessment Service. (2020).

6 Samms-Vaughan, M. E. The status of early identification and early intervention in autism spectrum disorders in lower- and middle-income countries. International Journal of Speech-Language Pathology 16, 30-35, doi:10.3109/17549507.2013.866271 (2014).

7 Wang, K., Wang, C., Guo, D., van Wijngaarden, M. \& Begeer, S. Children with autism spectrum disorder from China and the Netherlands: Age of diagnosis, gender and comorbidities. Research in Autism Spectrum Disorders 54, 76-82, doi:https://doi.org/10.1016/j.rasd.2018.07.004 (2018).

8 Tsang, K. M. S. et al. JC A-Connect: Jockey Club Autism Support Network Family Support Programme Final Report. 167 (The University of Hong Kong, Hong Kong, 2017).

9 Ferraioli, S. J. \& Harris, S. L. Comparative Effects of Mindfulness and Skills-Based Parent Training Programs for Parents of Children with Autism: Feasibility and Preliminary Outcome Data. Mindfulness 4, 89-101, doi:10.1007/s12671-012-0099-0 (2013).

10 Koegel, L. K., Koegel, R. L., Ashbaugh, K. \& Bradshaw, J. The importance of early identification and intervention for children with or at risk for autism spectrum disorders. International Journal of SpeechLanguage Pathology 16, 50-56, doi:10.3109/17549507.2013.861511 (2014).

11 Leung, C., Fan, A. \& Sanders, M. R. The effectiveness of a Group Triple P with Chinese parents who have a child with developmental disabilities: A randomized controlled trial. Research in Developmental Disabilities 34, 976-984, doi:https://doi.org/10.1016/j.ridd.2012.11.023 (2013).

12 World Health Organization. Autism Spectrum Disorders Fact Sheet, <http://www.who.int/mediacentre/factsheets/autism-spectrum-disorders/en/> (2017a).

13 Oono, I. P., Honey, E. J. \& McConachie, H. Parent-mediated early intervention for young children with autism spectrum disorders (ASD). Evidence-Based Child Health: A Cochrane Review Journal 8, 23802479, doi:10.1002/ebch.1952 (2013). 
14 Reichow, B., Barton, E. E., Boyd, B. A. \& Hume, K. Early Intensive Behavioral Intervention (EIBI) for Young Children with Autism Spectrum Disorders (ASD): A Systematic Review. Campbell Systematic Reviews 10, 1-116, doi:10.4073/csr.2014.9 (2014).

15 Reichow, B., Volkmar, F. R. \& Bloch, M. H. Systematic Review and Meta-analysis of Pharmacological Treatment of the Symptoms of Attention-Deficit/Hyperactivity Disorder in Children with Pervasive Developmental Disorders. Journal of Autism and Developmental Disorders 43, 2435-2441, doi:10.1007/s10803-013-1793-z (2013).

16 Salomone, E. et al. Development of the WHO Caregiver Skills Training Program for Developmental Disorders or Delays. Frontiers in Psychiatry 10, doi:10.3389/fpsyt.2019.00769 (2019).

17 WHO. Adaptation and Implementation Guide of the Parent Skills Training Programme for Families with Children with Developmental Disorders and Delays. (2015).

18 WHO. WHO Parent Skills Training Programme for Caregivers of Children with Deveopmental Disorders: Monitoring and Evaluation Framework. (2016).

19 Hsieh, H.-F. \& Shannon, S. E. Three Approaches to Qualitative Content Analysis. Qualitative Health Research 15, 1277-1288, doi:10.1177/1049732305276687 (2005).

20 Graneheim, U. H. \& Lundman, B. Qualitative content analysis in nursing research: concepts, procedures and measures to achieve trustworthiness. Nurse Education Today 24, 105-112 (2004).

21 Durkin, M. S. et al. Evaluating a ten questions screen for childhood disability: reliability and internal structure in different cultures. Journal of Clinical Epidemiology 48, 657-666 (1995).

22 Kasari, C. et al. Caregiver-mediated intervention for low-resourced preschoolers with autism: an RCT. Pediatrics 134, e72-e79 (2014).

23 Miles, M. B. \& Huberman, A. M. Qualitative data analysis: $A$ sourcebook of new methods. (Sage publications, 1984).

24 The Government of the Hong Kong Special Administrative Region. Prevention and Control of Disease (Prohibition on Group Gathering) Regulation. Government Press (2020).

25 Sun, K. S. et al. Barriers of Chinese primary care attenders to seeking help for psychological distress in Hong Kong. Journal of Affective Disorders 196, 164-170, doi:https://doi.org/10.1016/j.jad.2016.02.041 (2016).

26 Mali, J. The method of personal planning and the implementation of services in social work with older people. Ljetopis socijalnog rada 26, 61-80 (2019). 
27 Fabrizio, C. S., Lam, T. H., Hirschmann, M. R. \& Stewart, S. M. A Brief Parenting Intervention to Enhance the Parent-Child Relationship in Hong Kong: Harmony@Home. Journal of Child and Family Studies 22, 603-613, doi:10.1007/s10826-012-9614-0 (2013).

28 Stewart, S. M., Fabrizio, C. S., Hirschmann, M. R. \& Lam, T. H. Developing community-based preventive interventions in Hong Kong: a description of the first phase of the family project. BMC Public Health 12, 106, doi:10.1186/1471-2458-12-106 (2012).

29 Schultz, T. R., Schmidt, C. T. \& Stichter, J. P. A Review of Parent Education Programs for Parents of Children With Autism Spectrum Disorders. Focus on Autism and Other Developmental Disabilities 26, 96104, doi:10.1177/1088357610397346 (2011).

30 Stahmer, A. C. et al. Project ImPACT for Toddlers: Pilot outcomes of a community adaptation of an intervention for autism risk. Autism 24, 617-632, doi:10.1177/1362361319878080 (2020).

\section{Tables}

Table 1. Descriptive characteristics of caregivers and children in need assessment focus groups $(n=15)$ 
Nationality

Chinese

$12 \quad 80.0$

Nepal

320.0

Relationship with child

Mother

$15 \quad 100$

Age, $M(S D)$, range

38.1

(2.2),

34-41

\section{Educational attainment}

Secondary education

$6 \quad 40.0$

Tertiary education or above

$9 \quad 60.0$

Monthly household income (HKD)

$\$ 10,000$ to $\$ 24,999$

$6 \quad 40.0$

$\$ 25,000$ to $\$ 39,999$

$\begin{array}{ll}4 & 26.7\end{array}$

$\$ 40,000$ to $\$ 59,999$

$1 \quad 6.7$

$>\$ 56,000$

$\begin{array}{ll}4 & 26.7\end{array}$

Employment status

Part-time employed

17.0

Home-makers or unemployed

$14 \quad 93.0$

Marital status

Married

$15 \quad 100$

Year(s) living with the target child

Since birth

$14 \quad 93.3$

5 years

$\begin{array}{ll}1 & 6.7\end{array}$

Types of family

Nuclear family

$14 \quad 93.3$

Extended family

$1 \quad 6.7$

Number of children per family 1

$6 \quad 40.0$

2

$9 \quad 60.0$

Order of the target child

$1^{\text {st }}$

$\begin{array}{ll}10 & 66.7\end{array}$

$2^{\text {nd }}$

$\begin{array}{ll}5 & 33.3\end{array}$

Living district 
Shatin

Wong Tai Sin

320.0

Kowloon city

320.0

Eastern

$2 \quad 13.3$

Kwun Tong

$\begin{array}{ll}1 & 6.7\end{array}$

Yau Tsim Mong

$\begin{array}{ll}1 & 6.7\end{array}$

Types of training programs that had participated in before $\left(\mathrm{n}=12^{\mathrm{a}}\right)$

PACT

Happy Parenting

$2 \quad 13.3$

Louis Program

$2 \quad 13.3$

1

6.7

Educational talk on social communication skills, language development

$1 \quad 6.7$

Others: ABA, More Than Words Workshop (The Hanen Program), SCERTS model

7

SEN certificate course, executive function, social skills training, mindfulness 


\begin{tabular}{|c|c|c|c|}
\hline Child's characteristics & \multicolumn{2}{|c|}{$n$} & \\
\hline \multicolumn{4}{|l|}{ Gender } \\
\hline Male & 13 & & 86.7 \\
\hline Female & 2 & & 13.3 \\
\hline Child's age, $M(S D)$, range & \multicolumn{3}{|c|}{$6.2(1.6), 3-9$} \\
\hline \multicolumn{4}{|l|}{ Educational attainment } \\
\hline Nursery & 1 & & 6.7 \\
\hline Kindergarten & 2 & & 13.3 \\
\hline Primary schools & 5 & & 33.3 \\
\hline Special schools & 7 & & 46.7 \\
\hline \multicolumn{4}{|l|}{ Diagnosis } \\
\hline ASD only & 6 & & 40.0 \\
\hline $\mathrm{ASD}+\mathrm{ADHD}$ & 5 & & 33.3 \\
\hline ASD + language delays & 3 & & 20.0 \\
\hline ASD + genetic disorders & 1 & & 6.7 \\
\hline Year(s) of diagnosis & \multicolumn{3}{|c|}{$3.0(1.5)$} \\
\hline \multicolumn{4}{|c|}{ Received service provided by CACs under DH } \\
\hline Yes & 3 & \multicolumn{2}{|c|}{20.0} \\
\hline No & 12 & \multicolumn{2}{|l|}{80.0} \\
\hline \multicolumn{4}{|c|}{ Received service provided by EETCs } \\
\hline Yes & 3 & \multicolumn{2}{|l|}{20.0} \\
\hline No & 12 & \multicolumn{2}{|c|}{80.0} \\
\hline
\end{tabular}

Received service provided by IP in kindergarten-cum-childcare-centres

$\begin{array}{lll}\text { Yes } & 1 & 6.7 \\ \text { No } & 14 & 93.3\end{array}$

Received service provided by OPRS

$\begin{array}{lll}\text { Yes } & 4 & 26.7 \\ \text { No } & 11 & 73.3\end{array}$

Received service provided by SCCC

$\begin{array}{lll}\text { Yes } & 6 & 40.0 \\ \text { No } & 9 & 60.0\end{array}$

Received service provided by HA

$\begin{array}{lll}\text { Yes, Child and Adolescent Psychiatric Service } & 1 & 6.7\end{array}$

Yes, Step Center for Child Development under DKCH 2213.3

$\begin{array}{lll}\text { No } & 12 & 80.0\end{array}$ 


\begin{tabular}{lcc}
\hline Number of NGO service(s) received & & \\
None & 5 & 33.3 \\
1 & 2 & 13.3 \\
2 & 6 & 40.0 \\
\hline
\end{tabular}

Note. $n=$ number of participants; $M=$ mean; $S D=$ standard deviation; ABA $=$ Applied Behavior Analysis; HKD = Hong Kong Dollars; PACT = Pre-school Autism Communication Trial; SCERTS = Social Communication, Emotional Regulation, Transactional Support; SEN = Special Education Needs. ${ }^{a} 12$ out of 15 interviewed caregivers attended at least 1 training program in their lifetime. Of these, 7 attended more than 1 type of training program. Note. $M=$ mean; SD = standard deviation; HKD = Hong Kong Dollars; ADHD = Attention Deficient Hyperactive Disorder; ASD = Autism Spectrum Disorder; CAC $=$ Child Assessment Center; $\mathrm{DH}=$ Department of Health; DKCH $=$ Duchess of Kent Children's Hospital; EETC $=$ Early Education and Training Centre; HA = Hospital Authority; IP = integrated program; OPRS = On-site Preschool Rehabilitation Services; NGO = non-governmental organization; SCCC $=$ Special Child Care Center.

Table 2- Most mentioned items in focus group interviews with parents

\begin{tabular}{llc}
\hline Most mentioned items & $\mathrm{n}$ \\
\hline Pre-CST & Uncooperative family member & 22 \\
& Understand and manage child's inappropriate behavior & 18 \\
& Train up children's communication skills & 16 \\
Child being uncooperative during home practice & 15 \\
Train up children's social skills & 13 \\
& Learn skills that are relevant to their actual situations & 13 \\
\hline Post-CST & Overall reduction in challenging situation & 25 \\
& Improve on caregiving skills & 24 \\
& Manage own emotion as a caregiver and to take better care of themselves & 20 \\
& Home visit as useful element & 19 \\
& Understand and manage child's inappropriate behaviors & 16 \\
\hline
\end{tabular}


Figures

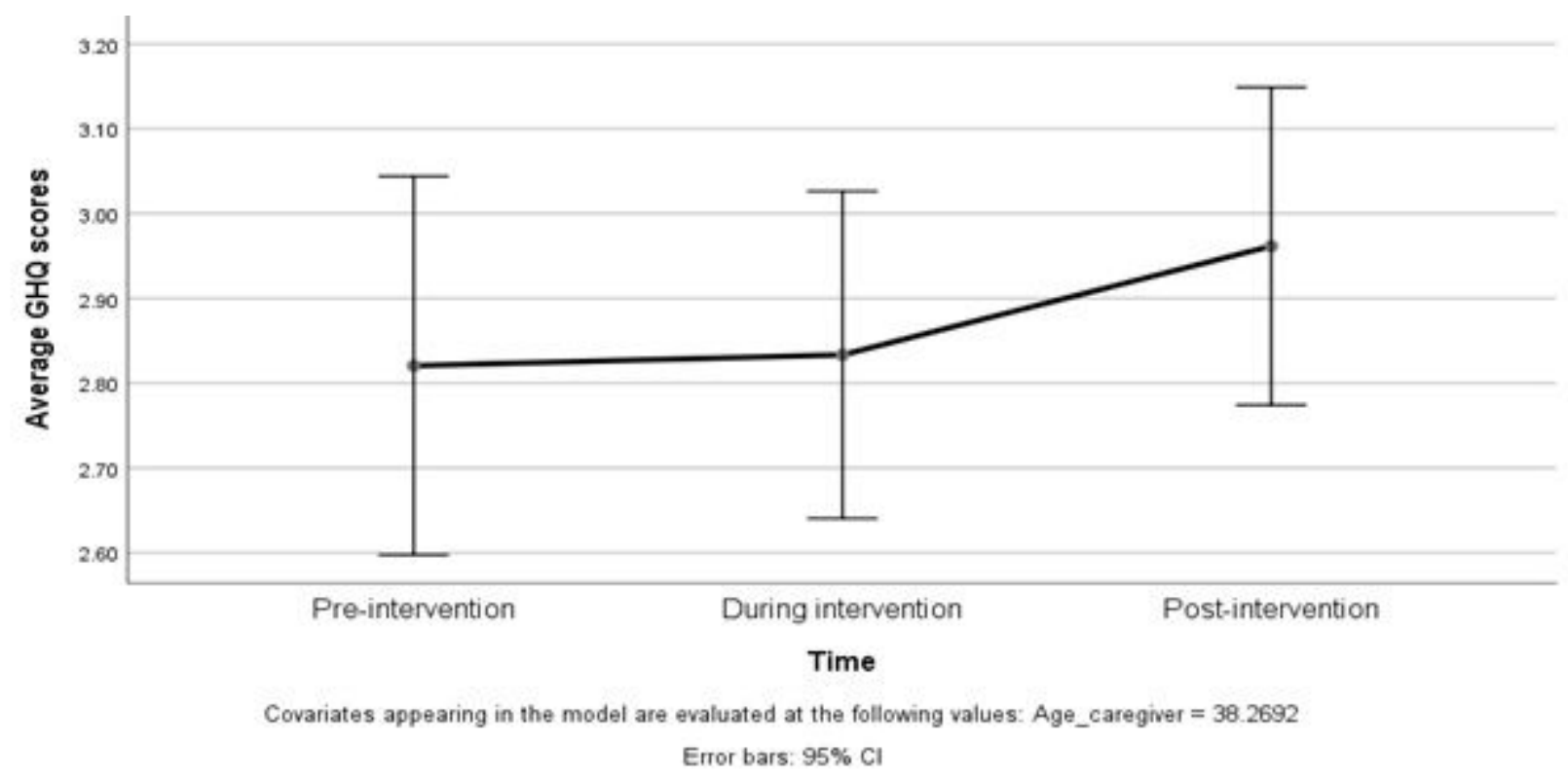

\section{Figure 1}

The average GHQ scores during three measurement periods

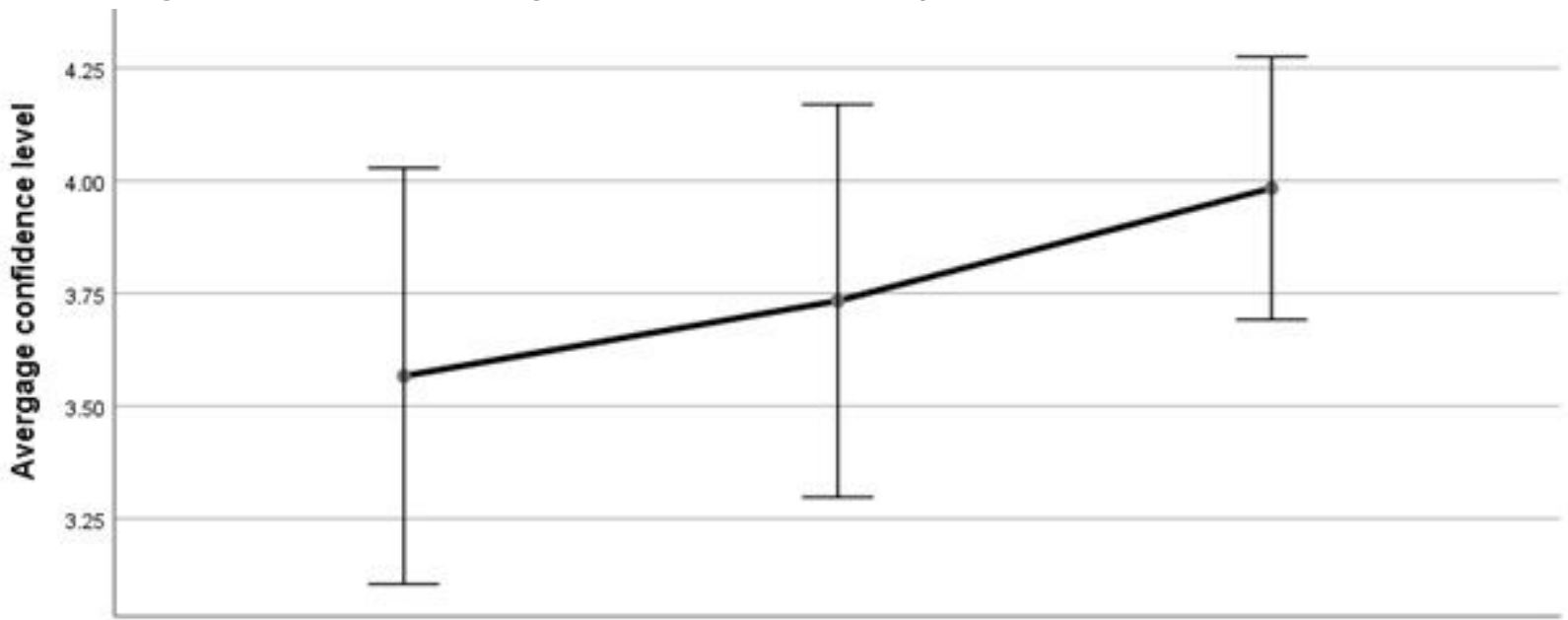

Pre-intervention

During intervention

Post-intervention

\section{Time}

Covariates appearing in the model ate evaluated at the following values: Age_caregiver $=38.1250$

Error bars: $95 \% \mathrm{Cl}$

\section{Figure 2}

The average confidence scores during three measurement periods 